\title{
Perceptual invariance for stop consonants in different positions
}

\author{
CATHERINE G. WOLF \\ Massachusetts Institute of Technology, Cambridge, Massachusetts 02139
}

\begin{abstract}
The basis for the invariant perception of place of articulation in pre- and postvocalic stops was investigated using the selective adaptation paradigm. Experiments 1 and 2 considered the role of identical bursts, mirror-image formant transitions, and similar onset and offset spectra in the invariant perception of place of articulation in CV and VC stimuli, and Experiment 3 considered the importance of the second two cues in a VCV context. The results of these experiments suggest that, at the level of processing tapped by selective adaptation, neither identical bursts, mirror-image formant transitions, nor similar onset and offset spectra are the basis for the invariant perception of place of articulation in initial and final position. The vowel portion of an adapter was found to affect perception of the consonant portion of a stimulus, and the direction of this effect was predictable from the acoustic characteristics of the consonant and vowel. The implications of these findings for the nature of selective adaptation are discussed.
\end{abstract}

One of the most interesting questions for the study of speech perception is the question of how listeners perceive the same phonetic segment in different contexts when the acoustic correlates of the segments are not the same. This question is particularly relevant to the perception of the place of articulation of stop consonants, since the acoustic cues to place of articulation appear to vary with the vowel context and syllabic position (Liberman, Cooper, Shankweiler, \& Studdert-Kennedy, 1967). ${ }^{1}$

It is well accepted that the transitions of the second (F2) and third (F3) formants are important cues to place of articulation of stop consonants (Delattre, Liberman, \& Cooper, 1955; Liberman et al., 1967). However, the formant transitions for a particular consonant vary with the adjacent vowel and with the position of the consonant relative to the vowel. Consider, for instance, the often-cited example that the second-formant transition necessary for the perception of [d] in a two-formant synthetic [di] differs in direction and frequency range from the secondformant transition necessary for [d] in [du] (Liberman, et al., 1967). When the variable syllable position is considered, one finds that the directions of the formant transitions (i.e., rising or falling in frequency over time) for a particular place of articulation are opposite in pre- and postvocalic stops.

The author is currently at Bell Labs, Holmdel, New Jersey 07733. This research was conducted while the author was a postdoctoral fellow at the Research Laboratory of Electronics, M.I.T., and was supported by NINCDS Postdoctoral Fellowship 1 F22 NS00796-01 to the author and by NINCDS Grant NS04332. The author would like to thank K. N. Stevens, J. L. Miller, W. F. Ganong, and two anonymous reviewers for their helpful comments on the manuscript, and T. Smith and L. Lampron for testing some of the subjects.
That is, initial formant transitions tend to mirror final formant transitions over time. The mirrorimage nature of initial and final formant transitions is apparent in the spectrogram of the synthetic stimuli in Figure 1.

The research to be reported investigated the basis for the invariant perception of place of articulation in different syllable positions using the selective adaptation paradigm. With the selective adaptation procedure (initially applied to speech by Eimas and Corbit, 1973), the identification of a series of synthetic speech sounds is assessed under two conditions. In one condition, unadapted identification, the sounds are simply presented in random order for identification. In the other condition, adapted identification, each sound to be identified is preceded by a minute or so of repeated presentation of an adapting sound, often one of the endpoint stimuli of the series. Adaptation is said to occur when repeated presentation of the adapter changes subjects' identification of the series of synthetic speech sounds relative to unadapted identification. In Eimas and Corbit's (1973) study, this change was measured as a shift in the phonetic boundary of the test series; more recently, changes in the identification of sounds near the endpoints of the series have also been reported (Miller, 1975; Sawusch, 1976). When the adapter is an endpoint stimulus of the series, identification of the sounds typically changes so that the phonetic boundary is shifted in the direction of the adapter.

On the basis of such findings, it has been proposed that the perception of phonetic segments is mediated by detectors which are sensitive to the features underlying various phonetic distinctions. ${ }^{2}$ Repeated presentation of an adapting sound decreases the 


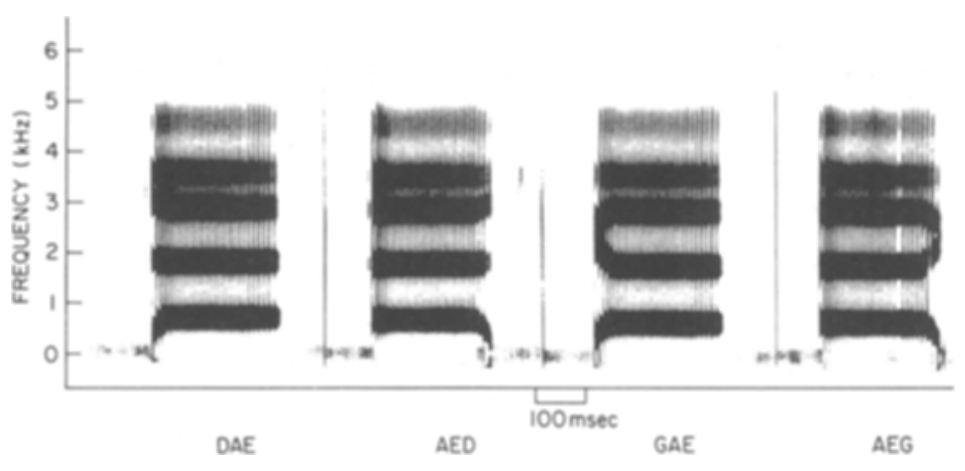

Figure 1. Spectrograms of the adapters for Experiment 1 are shown.

sensitivity, and, consequently, output, of detectors which are sensitive to the particular characteristics of that sound. For example, given a [bae]-[dae] test series, adaptation with [bae] decreases the output of detectors sensitive to the characteristics of [bae] relative to the output of detectors sensitive to the characteristics of [dae] (Cooper, 1974). A shift in the $[\mathrm{b}]-[\mathrm{d}]$ phonetic boundary results, since identification of the consonant depends on the relative output of detectors for the characteristics of both [bae] and [dae]. Subsequent studies have reported similar adaptation effects for other phonetic distinctions (see Ades, 1976, and Cooper, 1975, for reviews of the literature).

It is obvious that, in order to perceive the phonetic identity of initial and final stops, listeners must process some aspect of initial and final stops identically at some level of processing. The selective adaptation paradigm can be a useful tool in the investigation of the aspects of pre- and postvocalic stops which are responsible for the invariant perception of place of articulation across syllable position. Ades (1974) used the selective adaptation procedure to study the role of mirror-image formant transitions in the perception of the place of articulation of initial and final stops by assessing the effects of a vowelconsonant (VC) adapter on a consonant-vowel (CV) continuum, and vice versa. Specifically, two synthetic speech series, [bae]-[dae] and [aeb]-[aed], were constructed in which the formant transitions of the CVs and VCs were mirror images. The endpoints of each series were used as adapters on the other series (cross-series condition) and also on the series to which they belonged (within-series condition). Ades found that the cross-series adapters did not affect the phonetic boundary in the same way as the within-series adapters. The only effect for the cross-series adapters was a small [d]-like effect for all cross-series conditions. Ades (1974) thus concluded that "there are different detectors for consonants in initial and final position." Later adaptation studies (Miller \& Eimas, 1976; Sawusch, Note 1) also failed to find a cross-series effect.
Ades' (1974) conclusion that initial and final consonants are processed by different detectors may have been somewhat premature. Formant transitions, while extremely important cues to place of articulation, are not the only place cues for stop consonants. There is ample evidence that the brief noise burst which occurs at the release of closure is an important cue to place of articulation for initial stops (Minifie, Rudegair, Milstein, \& Vivion, 1971; Bush $\&$ Williams, Note 2; Fischer-Jorgensen, Note 3), and can be even more important than formant transitions in final position (Malecot, 1958; Wang, 1959). Since the burst for an initial consonant may be similar to the burst for the same consonant in final position (Halle, Hughes, \& Radley, 1956), the burst might serve as the basis for the invariant perception of place of articulation in initial and final consonants.

Experiment 1 investigated the role of the burst in the perception of place of articulation in initial and final stops using the selective adaptation paradigm. Previous work has shown that bursts are processed at the level affected by selective adaptation (Blumstein, Stevens, \& Nigro, 1977; Diehl, 1975). In Experiment 1, synthetic CV and VC series were constructed in which place of articulation was cued by the burst as well as the second- and third-formant transitions. The burst was identical for a consonant in initial and final positions, and the formant transitions were mirror images. A burst in one position might affect the perception of the place of articulation of a consonant in the other position if there are detectors tuned to a noise burst with a particular frequency spectrum regardless of its position within a syllable.

Experiment 2 followed up some interesting findings of Experiment 1 by examining the adaptation effect of the vowel portion of the adapter on the consonant portion of the test stimuli, and Experiment 3 investigated the perception of initial and final place cues in a VCV context.

The experiments reported in this paper were premised on the assumption that (at least some) auditory processes can be tapped by the selective 
adaptation paradigm. Thus, the study sought to find the auditory basis for the perceptual invariance of place cues in different positions using selective adaptation. It should be noted that these experiments were not intended to address the issue of the auditory vs. phonetic basis of selective adaptation.

\section{EXPERIMENT 1}

\section{Method}

Subjects

Two groups of eight paid subjects were used. The subjects were M. I. T. students and employees and were all native speakers of American English with no known hearing impairment.

Stimuli

The stimuli were five-formant synthetic speech sounds produced with Klatt's (1972) terminal-analog speech synthesis program. Two series of stimuli were generated: a VC [aed]-[aeg] series and a $\mathrm{CV}$ [dae]-[gae] series. The member of a series differed from each other in the F2 and F3 transitions and the burst. The two endpoint stimui of each series served as adapters. Figure 1 shows spectrograms of the four adapters.

The VC stimuli consisted of a $270-\mathrm{msec}$ formant structure, followed by a closure interval of 50 or $60 \mathrm{msec}$, which was followed by a $10-\mathrm{msec}$ noise burst. The steady-state values of the formants were $650 \mathrm{~Hz}$ for $F 1,1,750 \mathrm{~Hz}$ for $F 2,2,800 \mathrm{~Hz}$ for F3, $3,500 \mathrm{~Hz}$ for $\mathrm{F} 4$, and $4,500 \mathrm{~Hz}$ for F5. The first formant had a 35-msec transition with an ending frequency of $225 \mathrm{~Hz}$ for all stumuli. The second formant transition was $40 \mathrm{msec}$ long and had an ending frequency of $1,800 \mathrm{~Hz}$ for the extreme [aed] stimulus (Stimulus 0) and $2,224 \mathrm{~Hz}$ for the extreme [aeg] stimulus (Stimulus 8). The F3 transition was also $40 \mathrm{msec}$ long and had endpoints of $2,974 \mathrm{~Hz}$ for Stimulus 0 and $2,550 \mathrm{~Hz}$ for Stimulus 8 . The F2 and F3 transition ending frequencies for the nine stimuli of the series varied in $53-\mathrm{Hz}$ steps from the extreme [aed] to the extreme [aeg] stimulus.

The extreme [aed] stimulus had a 10-msec noise burst centered at the frequency of $F 4$, which occurred $50 \mathrm{msec}$ after the end of the formant structure. The extreme [aeg] stimulus had a 10-msec burst, centered at F3, which occurred $60 \mathrm{msec}$ after the end of the formant structure. The burst for the stimuli between the [aed] and [aeg] endpoints was produced by decreasing the amplitude of the F4 burst from its maximum to zero in 7-dB steps between Stimulus 0 and Stimulus 8 , and increasing the amplitude of the F3 burst from zero to its maximum between Stimuli 0 and 8 . The effective closure duration, thus, was either 50 or $60 \mathrm{msec}^{3}$ Low-frequency periodic energy simulating glottal pulsing was present throughout the closure interval.

The fundamental frequency rose from 103 to $112 \mathrm{~Hz}$ over the first $35 \mathrm{msec}$ of the stimuli and fell to $90 \mathrm{~Hz}$ over the last $120 \mathrm{msec}$ of the formant structure. The overall amplitude of the stimuli rose to its peak level gradually over the first $20 \mathrm{msec}$.

The formant structure of the [dae]-[gae] stimuli was the mirror image of the [aed]-[aeg] stimuli. The bursts were identical to those of the VC series. The [dae] burst was located immediately before the onset of the formant structure and the [gae] burst began $20 \mathrm{msec}$ before the onset of the formant structure. Thus, there was a 10-msec gap between the end of the [gae] burst and the onset of the formant structure. The fundamental frequency contour was the same as for the VC series, and the overall amplitude rose and fell gradually at the onset and offset of the formant structure.

The stimuli were recorded on a two-channel Presto 800 tape recorder.

\section{Design and Procedure}

There were four sets of conditions: (1) the CV stimulus series with the [dae] and [aed] adapters, (2) the VC stimulus series with the [dael and [aed] adapters, (3) the CV stimulus series with the [gae] and [aeg] adapters, and (4) the VC stimulus series with the [gae] and [aeg] adapters. One group of eight subjects was given the first two sets of conditions, and the other group of eight was given the conditions described in (3) and (4).

Construction of the stimulus tapes. Two types of tapes were generated: unadapted identification tapes and adapted identification tapes. For each series of stimuli, three unadapted and four adapted identification tapes were made. Each unadapted identification tape consisted of a practice block of four of each of the nine stimuli in random order, followed by two blocks of eight of each of the stimuli. There was a $3-\mathrm{sec}$ pause after every trial, with an additional $2-\mathrm{sec}$ pause after every 10th trial.

Each set of four adaptation trials consisted of: (1) 45 presentations of the adapter, with a $250-\mathrm{msec}$ interval between repetitions; (2) a 1.25 -sec interval before presentation of the first stimulus to be identified; and (3) presentation of the four test stimuli with a 3-sec interval after each stimulus. Each adapted identification tape consisted of a set of four practice trials, followed by two blocks of eight presentations of each of the nine stimuli (i.e., 36 sets of four trials). The same adapter was used throughout a tape.

Testing procedure. The experiment was administered in five sessions of about $1 \mathrm{~h}$ each on consecutive (or nearly consecutive) days. On the first day, which was used as practice, the subjects listened to two unadapted identification tapes of both the $\mathrm{CV}$ and VC stimuli. The subjects were asked to identify the stimuli using the 8-point rating scale shown in Table 1. A rating scale was used because this response measure has been shown to be more sensitive to adaptation effects in the vicinity of the endpoints of a series than the conventional phoneme labeling response (Sawusch, 1976). On Days 2 through 5, the subjects were first given an unadapted identification tape and then an adapted identification tape. The subjects again identified the stimuli using the 8-point rating scale. The tapes were played on a two-channel Revox A77 and subjects listened to the tapes over Sharpe HA 660/PRO headphones.

\section{Results \\ Effects of the Adapters on the Stimulus Series}

Figure 2 presents the average rating responses to the nine stimuli of a series in the unadapted identification condition and four sets of adapted identification conditions.

In order to assess the effects of the adapters on identification of the stimuli, a separate analysis of variance was done for the factors adapter (VC, $\mathrm{CV}$, or none) and stimulus (0 through 8) for each of the four sets of conditions. In this way, the effects of the $\mathrm{CV}$ and VC adapters could be compared with each other and with the unadapted identification condition. In all analyses, the mean unadapted iden-

Table 1

Rating Scale Used in Experiments 1 and 2

1. A very definite "d,"

2.

3.

4. Not at all definite: best guess is "d."

5. Not at all definite: best guess is "g."

6.

7.

8. A very definite "g." 

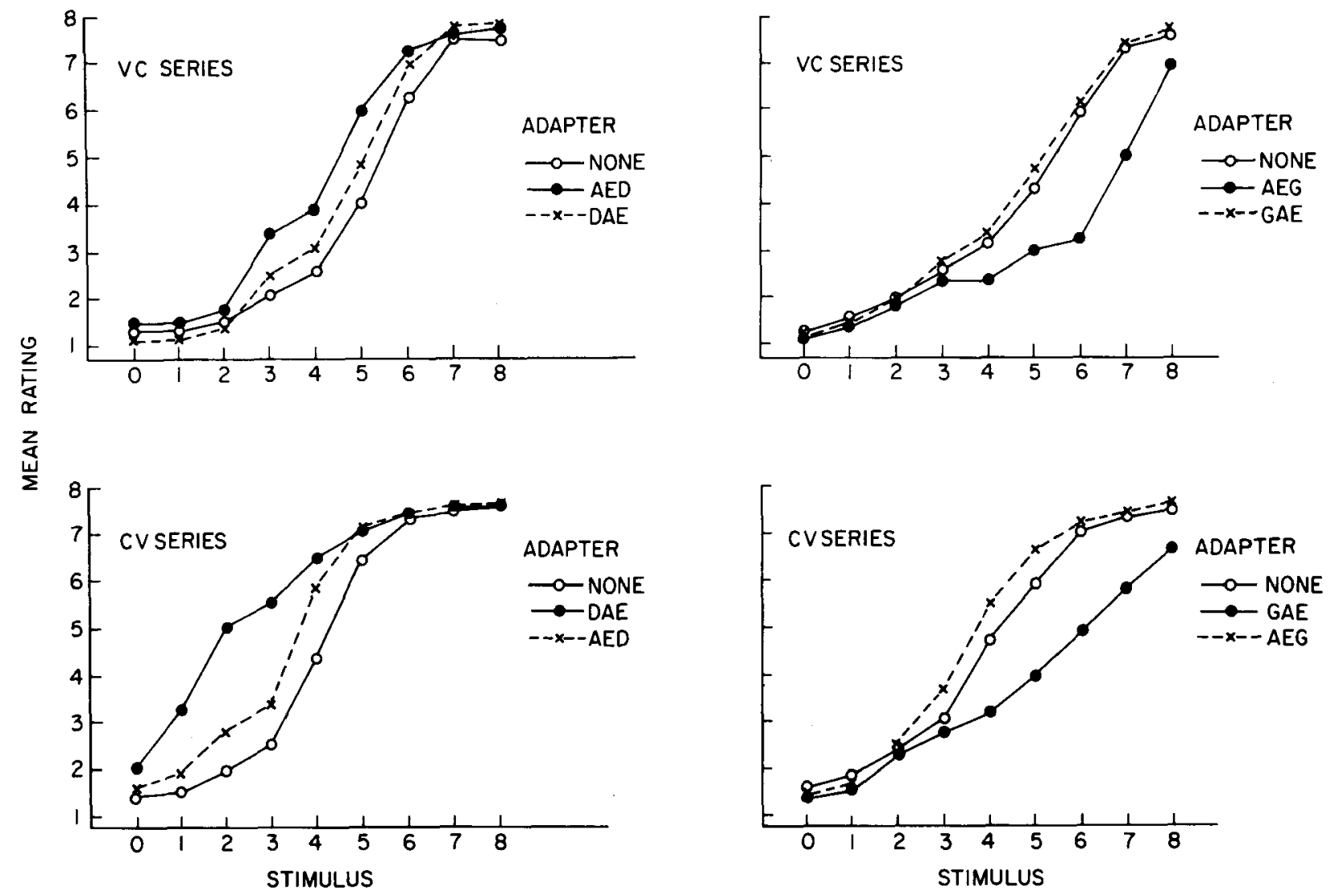

Figure 2. Mean ratings in each of the four sets of adaptation conditions are shown as a function of the stimulus for Experiment 1. On all graphs, the unadapted identification condition is represented by the unfilled circles, the within-series condition by the filled circles, and the cross-series condition by the crosses.

tification rating was based on the two sessions in which a subject was tested on a particular stimulus series.

The analysis of variance for the $\mathrm{CV}$ series with the [d] adapters indicated the following effects to be significant: adapter, $F(2,14)=46.80$, stimulus, $F(8,56)=125.03$, and the Adapter by Stimulus interaction, $F(16,112)=12.35, p<.001$ in all cases. Post hoc analyses of the difference between means (Lindquist, 1953) for the adapter factor revealed that both the [dae] and [aed] adapters moved the average rating toward the " $G$ " end of the scale, compared with the average rating in the unadapted identification condition, $\mathrm{p}<.001$. In addition, the mean rating with the [dae] adapter was significantly higher (i.e., more "G"-like) than with the [aed] adapter, $\mathrm{p}<.001$. Post hoc tests of the interaction indicated that the average rating with the [dae] adapter was higher than in the unadapted identification condition for Stimuli 0 through 5; with the [aed] adapter, the average rating was significantly higher than for the unadapted identification condition for Stimuli 1 through $5, \mathrm{p}<.05$ or more in all cases. The rating with the [dae] adapter was also significantly higher than with the [aed] adapter for Stimuli 2, 3, and 4, $\mathrm{p}<.05$ or more. These differences are apparent in the graph in the bottom left corner of Figure 2: over the first five or six stimuli the [dae] function tends to lie above the [aed] function, which tends to lie above the unadapted identification function.

The analysis of variance for the $\mathrm{CV}$ series with the [g] adapters revealed a somewhat different picture. The factors adapter, $F(2,14)=47.31$, stimulus, $F(8,56)=240.04$, and the Adapter by Stimulus interaction, $F(16,112)=10.31$, were all highly significant $(p<.001)$. Post hoc analysis of the difference between means for the adapter factor revealed that the average rating with the [gae] adapter was significantly lower (more " $D$ "'-like) than with no adapter, $\mathrm{p}<.001$, but the average rating with the [aeg] adapter was marginally higher (more " $G$ "-like) than with no adapter $(p<.10)$. Analysis of the interaction factor indicated that the mean rating with the [gae] adapter was significantly lower than the mean rating with no adapter for Stimuli 4 through $8, \mathrm{p}<.05$ or more. The [aeg] adapter significantly increased the average rating over that in the unadapted identification condition for the 
stimuli in the middle of the series (Stimuli 3, 4, and 5 ), $p<.05$ or better in all cases.

The analysis of variance for the [d] adapters with the VC series indicated statistical significance for the factors adapter, $F(2,14)=29.13$, stimulus, $F(8,56)=140.13$, and the Adapter by Stimulus interaction, $F(16,112)=6.94, \quad p<.001$. The post hoc tests of the difference between means for the adapter factor revealed that both the [dae] $(p<.05)$ and [aed] $(p<.001)$ adapters produced a higher average rating than the unadapted identification condition, and that the rating with [aed] was significantly higher than the rating with [dae] $(p<.001)$. Examination of the interaction indicated that the mean rating with either the [aed] or [dae] adapter was statistically greater than with no adapter for Stimuli 3 through 6; $\mathrm{p}<.05$ or more. The [aed] adapter resulted in significantly higher ratings than the [dae] adapter for Stimuli 3 through 5.

For the VC series with the [g] adapters, the analysis of variance showed the following factors to be significant: adapter, $F(2,14)=119.39$, stimulus, $F(8,56)=315.01$, and the Adapter by Stimulus interaction, $F(16,112)=24.78$, all $p<.001$. The post hoc test of the difference between means for the adapter factor found that the mean rating with the [aeg] adapter was lower (more " $D$ "-like) than with no adapter, and also lower than with the [gae] adapter, $\mathrm{p}<.001$. No significant difference was found for the [gae] adapter compared with the unadapted identification condition. The post hoc tests of the interaction indicated the mean rating for the [aeg] adapter to be lower compared with no adapter for Stimuli 4 through 8; the [gae] adapter resulted in a significantly higher rating than for the unadapted identification condition for Stimulus 5, $\mathrm{p}<.05$ or more. The rating with the [aeg] adapter was significantly lower than with the [gae] adapter for Stimuli 3 through 8, $p<.05$ or more.

\section{Phonetic Boundaries}

Phonetic boundaries were determined for each subject for the adapted and unadapted identification conditions by linear interpolation between the two stimuli on either side of the boundary. ${ }^{4}$ One-way analyses of variance were performed on the boundaries for the adapter factor for each of the four sets of conditions. All $F$ ratios were significant at the .001 level. The mean phonetic boundaries are shown in Table 2.

For the CV stimulus series, post hoc tests of the difference between means indicated that the phonetic boundaries with the [dae] and [aed] adapters moved significantly towards the [d] end of the series compared with the unadapted identification condition ( $p<.01$ or more). The boundary with [dae] was also significantly different from that with [aed] $(p<.01)$.
The [gae] adapter significantly moved the phonetic boundary for the $\mathrm{CV}$ series towards the [g] end of the series $(p<.001)$, and the [aeg] adapter caused a significant shift toward the [d] end of the series $(p<.01)$. The boundaries with the [gae] and [aeg] adapters were, of course, significantly different from each other.

The phonetic boundaries for the VC series moved significantly towards the [d] end of the series with both [dae] $(p<.05)$ and [aed] $(p<.001)$; the boundary with [aed] was significantly closer to the [d] end of the series than with [dae] $(p<.01)$. The [aeg] adapter moved the phonetic boundary on the $\mathrm{VC}$ continuum significantly towards the $[\mathrm{g}]$ end $(p<.001)$. The [gae] adapter moved the boundary in the direction of the [d] end of the series, but this shift was not statistically significant. The boundaries with these two adapters were significantly different from each other $(p<.001)$.

\section{Comparison of $\mathrm{CV}$ and VC Unadapted Phonetic Boundaries}

It appears from Table 2 that the unadapted CV and VC phonetic boundaries are not the same; the CV boundary is about one stimulus unit closer to the [d] end of the series than the boundary for the VC series. A $t$ test confirmed this observation $[t(15)=$ $6.19, \mathrm{p}<.001]$. This observation is interesting because it suggests that the mapping from acoustic structure to phonetic percept is not identical for these CV and VC stimuli with identical bursts and mirror-image formant transitions.

\section{Discussion}

The expected within-series adaptation effects were found for all conditions in Experiment 1. In addition, the predicted cross-series effect was found for both [d] adapters. The [g] cross-series adapters, however, tended to have an effect opposite of that predicted. Clearly, these findings cannot be explained solely in terms of the effect of the burst. Reexamination of the second- and third-formant frequencies of the stimuli suggested that the cross-series [d]-like effect may have been due to the vowel portion of the adapters.

Table 2

Mean Phonetic Boundaries with the Different Adapters: Experiment 1

\begin{tabular}{ccc}
\hline Adapter & $\begin{array}{c}\text { CV Test Series } \\
\text { Boundary }\end{array}$ & $\begin{array}{c}\text { VC Test Series } \\
\text { Boundary }\end{array}$ \\
\hline None & 4.04 & 5.06 \\
[dae] & 1.82 & 4.69 \\
[aed] & 3.23 & 4.19 \\
None & 4.02 & 5.10 \\
[gae] & 5.64 & 4.91 \\
[aeg] & 3.45 & 6.80 \\
\hline
\end{tabular}


The steady-state F2 and F3 frequencies of the vowel were very close to the $F 2$ and $F 3$ transition frequencies for [dae] and [aed]. Thus, the initial portion of the [aed] and [aeg] adapters may have caused the adaptation effect on the CV stimuli and the final portion of the [dae] and [gae] adapters may have been responsible for the adaptation effect on the VC series. It should be noted that the cross-series [d]like adaptation effects were not as great as the within-series effects of [dae] and [aed]. This difference probably resulted because the critical portions of the cross-series adapters (especially F1) were less similar to the test stimuli than were the analogous portions of the within-series adapters.

Results such as these are not entirely without precedent. Ades' (1974) report of very slight [d]-like. effects for both his [d] and [b] cross-series adapters also could have been caused by the steady-state portion of his adapters.

The findings of Experiment 1, although quite interesting, do not answer the question originally motivating the experiment: Can the burst serve as the basis for the invariant perception of place of articulation in initial and final stops? It is quite possible that the cross-series adaptation effects were due to the vowel portion of the adapters. However, the possibility that the burst also had an effect cannot be ruled out. Given that the cross-series effects were somewhat greater for the [d] than for the [g] adapters, it is possible that the [d] burst added to the effect of the vowel, whereas the [g] burst worked in the opposite direction.

The second experiment was, therefore, designed to determine whether the burst had an adapting effect in addition to the effect of the vowel, or whether the cross-series [d]-like effects of Experiment 1 could be explained solely in terms of the vowel.

\section{EXPERIMENT 2}

Experiment 2 assessed the perception of the $\mathrm{CV}$ and VC stimuli of Experiment 1 with three types of adapters: a steady-state [ae], the cross-series [d] adapter of Experiment 1 ([dae] for the VC series, [aed] for the CV series), and the cross-series [d] adapters without the burst. This last adaptation condition was included to test the possibility that the formant transitions contributed to the adaptation effect in the cross-series conditions of Experiment 1. There are four conceivable outcomes to Experiment 2. (1) All the adapters may have the same effect on identification of the stimuli. One could then conclude that the cross-series effects of Experiment 1 were due solely to the vowel portion of the adapter. (2) The [d] adapter with burst may have a greater effect than the other adapters. If this occurs, it could be concluded that the burst in one position does affect perception of the consonant in the other position, and thus may play a role in the invariant perception of place in initial and final stops. (3) The two types of consonant adapters may produce an identical effect, but one which is greater than the effect of the [ae] adapter. This finding would indicate that the mirror-image formant transitions on a $\mathrm{CV}$ and $\mathrm{VC}$ affect the place of articulation of consonants in the other position, but that the burst has no effect. (4) It is also conceivable that the adaptation effects would be ordered such that: [d] adapter with burst $>$ [d] adapter without burst $>$ vowel alone. Such an ordering would suggest that both the formant transitions and burst contribute to the adaptation effect. Thus, the invariant perception of place in pre- and postvocalic stops could perhaps be explained by the fact that, at the level of selective adaptation, the same mechanisms process these cues in initial and final position.

\section{Method \\ Stimuli \\ The CV and VC stimuli of Experiment 1 were used as the test series. The extreme [d] stimulus in each series was used as the [d] adapter with burst; the [d] adapter without burst was made by deleting the burst and closure interval from the [d] adapter with burst, and the [ae] adapter was made by changing the F1, F2, and F3 transitions of the [d] adapters without burst to steady state.}

\section{Subjects}

Two groups of 11 M.I.T. students and employees served as subjects.

\section{Design and Procedure}

One group of 11 subjects was tested on the $\mathrm{CV}$ stimulus series with the three types of adapters, and the other group was tested on the VC series. The experiment was administered in four sessions with the first session consisting of unadapted identification practice only. All other design and procedural details were identical to Experiment 1.

\section{RESULTS \\ Effects of the Adapters on the Stimulus Series}

The mean ratings for the three adaptation conditions and the unadapted identification condition are shown for the CV and VC series in Figure 3. A separate two-way analysis of variance, Adapter ([ae], [d] with burst, [d] without burst, and none) by Stimulus ( 0 through 8 ) was done for each stimulus series. For the CV series, the analysis found significant effects for the adapter, $F(3,30)=7.53$, stimulus, $F(8,80)=115.36$, and the Adapter by Stimulus interaction, $F(24,240)=2.64, p<.001$ in all cases. The post hoc test of the difference between means for the adapter factor indicated that the average rating was significantly higher (more "G"-like) with each of the adapters compared with the unadapted identification condition $(\mathrm{p}<.05$ or more), and that there were no significant differences among the three adapters. The post hoc analyses of 


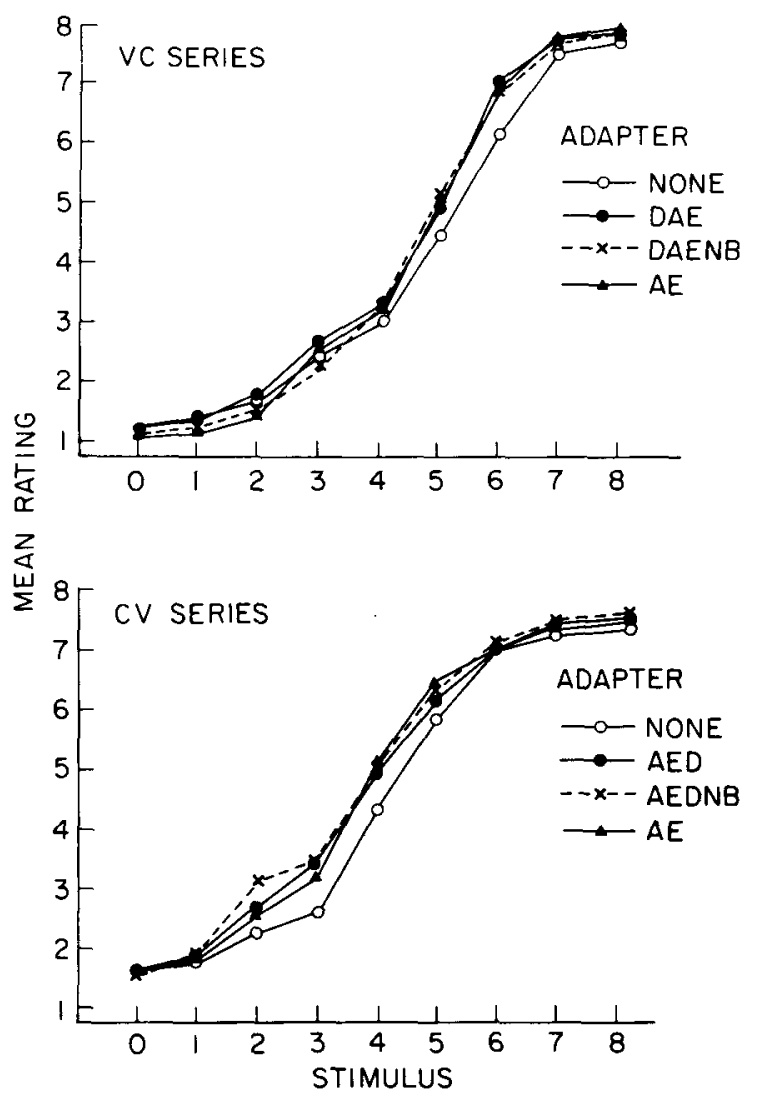

Figure 3. Mean ratings in the different adaptation conditions are shown as a function of the stimulus for Experiment 2. On both graphs, the unadapted identification condition is represented by the unfilled circles, the cross-series adapter with burst by the filled circles, the cross-series adapter without burst by the crosses, and the vowel adapter by the triangles.

the interaction revealed that the average rating with each of the three adapters was higher than with no adapter for Stimuli 2 through $5, \mathrm{p}<.05$ or more in all cases.

The analysis of variance for the VC series showed a significant effect of stimulus, $F(8,80)=312.63$, $\mathrm{p}<.001$, and a significant Adapter by Stimulus interaction, $F(24,240)=1.63, p<.05$. The post hoc tests of the difference between means for the interaction indicated that, for Stimulus 6, each of the adapters produced a rating which was higher than in the unadapted identification condition, $p<.05$ or more. For Stimulus 5 the mean ratings for the [dae] without burst and the [ae] were significantly higher than the rating with no adapter $(p<.05)$, and the mean rating for [dae] with burst was marginally higher than the unadapted identification, $\mathrm{p}<.10$. The only other statistically significant difference for the interaction was for Stimulus 3, where the [dae] adapter with burst produced a greater effect than the [dae] adapter without burst, $\mathrm{p}<.05$.

\section{Phonetic Boundaries}

Phonetic boundaries were determined as in Experiment 1, and are shown in Table 3. The oneway analysis of variance for the adapter factor was significant for the $\mathrm{CV}$ series, $\mathrm{F}(3,30)=4.06$, $\mathrm{p}<.05$. The phonetic boundaries with both [aed] adapters were significantly closer to the " $D$ " end of the scale than in the unadapted identification condition, $\mathrm{p}<.01$; for the [ae] adapter, the boundary was marginally closer to the " $D$ " end compared with the unadapted identification condition, $\mathrm{p}<.10$. There were no significant differences among the adapters. For the VC series, the $F$ ratio was not significant, although the difference between the mean rating in each of the adaptation conditions and the unadapted identification was greater than the differences among adapted identification conditions.

\section{Discussion}

The results for the $\mathrm{CV}$ test series failed to find evidence for the role of the burst or transitions in the invariant perception of place of articulation in initial and final position. Thus, the findings support the proposal that the [d]-like cross-series adaptation effects of Experiment 1 were caused by the vowel portion of the adapter. The adaptation effects for the VC test stimuli were smaller and somewhat less reliable than for the $\mathrm{CV}$ stimuli. This also tended to be true for the cross-series adapters in Experiment 1 .

There is a reasonable explanation for this difference between the VC and CV test stimuli based on the acoustic characteristics of the stimuli. In the VC stimuli, there was a 50 - or 60 -msec interval between the end of the formant structure and the burst, whereas in the CV test stimuli, the burst was followed almost immediately by the beginning of the formant structure. Because of these differences in the temporal structure of the $\mathrm{CV}$ and VC syllables, the similarity between the initial portion of the CV test stimuli and the VC or $\mathrm{V}$ adapters was greater than the similarity between the final portion of the VC test stimuli and the $\mathrm{CV}$ or $\mathrm{V}$ adapters. In terms of the selective adaptation process, in the former case there is greater overlap in the set of detectors in-

Table 3

Mean Phonetic Boundaries (B) with the Different Adapters: Experiment 2

\begin{tabular}{lclc}
\multicolumn{1}{c}{ CV Test Series } & \multicolumn{2}{c}{ VC Test Series } \\
Adapter & B & Adapter & B \\
\hline None & 4.23 & None & 4.99 \\
[aed] with burst & 3.65 & [dae] with burst & 4.74 \\
[aed] without burst & 3.73 & [dae] without burst & 4.67 \\
[ae] & 3.89 & [ae] & 4.71 \\
\hline
\end{tabular}


volved in the extraction of information from the adapters and test stimuli than in the latter.

At this point, it is appropriate to discuss the basis for the adaptation effects found in Experiments 1 and 2 and to relate these findings to other work. Sawusch (Note 1), in an extensive study of selective adaptation, reported findings which are relevant to an interpretation of the present results. In one experiment, a VC ([^d]) was used as an adapter on a CV test series ([bae]-[dae]) and a CV adapter ([bae]) was used on a VC test series $([\Lambda b]-[\Lambda d])$. The stimuli were designed so that the second- and third-formant transitions of the [bae] and $[\Lambda \mathrm{d}]$ endpoints were identical. As a consequence, the second and third formants of the steady-state $[\Lambda]$ were identical to the [bae] F2 and F3 transition starting points. No cross-series adaptation was found in these conditions. Another experiment failed to find an effect of either the isolated vowel $[\Lambda]$ or a vowellike sound in which F1, F2, and F3 were equal to the [bae] transition starting points. ${ }^{5}$

Sawusch's failure to find a true cross-position effect is in accord with the findings of the present study. The apparent discrepancy between the present finding of an adaptation effect caused by the vowel portion of an adapter and Sawusch's failure to find such an effect can be probably explained in terms of the acoustic characteristics of the stimuli in the two studies. Sawusch's test stimuli had steep formant transitions, with the consequence that the transition part of the test stimuli differed from the corresponding vowel portion of the adapter. In the present study, however, the entire second-and third-formant transitions of the test stimuli were similar to the corresponding portions of the adapter. This suggests that, in the present study, the adaptation produced by the vowel was based on the frequency characteristics of the vowel over the period of time corresponding more or less to the duration of the transition of the test stimuli, and not only to the frequency characteristics at the instant of onset (or offset) of the syllable.

The main findings of Experiments 1 and 2 can be explained in terms of either position-sensitive detectors that are tuned to the frequency spectrum of a stimulus in the vicinity of onset or offset or in terms of detectors tuned to specific formant transitions. However, the smaller cross-series adaptation effects on the VC continuum (which were explained on the basis of the contribution of the burst to consonant identification) can be more easily accounted for by a frequency spectrum detector hypothesis.

\section{EXPERIMENT 3}

The evidence seems to indicate that the position of place of articulation information relative to the vowel affects the way this information is processed. There appear to be separate detectors for place cues in different positions. These findings raise the question of whether or not initial and final place cues may be processed by the same detectors when the cues are embedded in a more similar context. Experiment 3 attempted to answer the question using vowel-consonant-vowel (VCV) stimuli. In natural speech, VCVs typically have transitions from the first vowel into the closure interval and from the closure interval into the second vowel. That is, VCVs contain both initial and final place cues. Unlike the place information in a $\mathrm{CV}$ or VC, however, the place information in a VCV is both preceded and followed by a vowel.

The test series in Experiment 3 consisted of a VCV continuum in which place of articulation was cued by formant transitions on the second vowel only. These stimuli may be considered to be "partial cue" stimuli (Blumstein et al., 1977). In natural speech, such stimuli might result if the first vowel terminated in a glottal stop. Two types of adapters were used. Partial cue adapters, which were the endpoints of the test series (referred to as A/BA and A/DA) and "full cue" adapters. The full cue adapters (referred to as ABBA and ADDA) were identical to the partial cue adapters except that they had the appropriate mirror-image formant transitions on the first vowel as well as the formant transitions on the second vowel (see Figure 4 for spectrograms for the four adapters).

Previous work has shown that full cue adapters

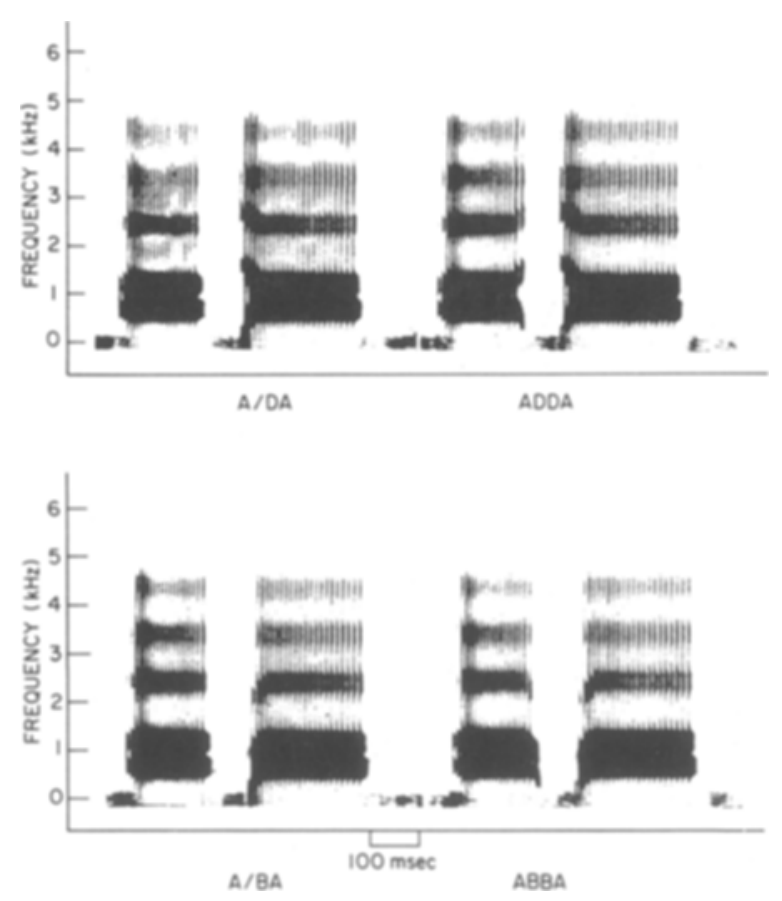

Figure 4. Spectrograms of the adapters for Experiment 3 are shown 
can have a greater adaptation effect than partial cue adapters (Blumstein et al., 1977). Therefore, if the same detectors process initial and final place of articulation cues in a VCV context, then the full cue adapters may have a greater effect than the partial cue adapters. There are at least two lines of reasoning which might argue for such an outcome. (1) The initial and final components of place of articulation information in a VCV may be processed by the same mechanism if the critical acoustic characteristic signaling place of articulation is the frequency spectrum in the vicinity of closure (Blumstein et al., 1977; Blumstein \& Stevens, Note 4; Stevens, Note 5). Experiments 1 and 2 indicate that different detectors may be tuned to the spectrum in the vicinity of onset and offset of consonants in initial and final position, respectively. However, the temporal window over which the spectrum is computed may be large enough so that, for a VCV, a detector centered on the onset of the $\mathrm{CV}$ portion also picks up information about the offset spectrum of the preceding VC, and vice versa for an offset detector. (2) Such a result could also be obtained if separate detectors sensitive to place information in intervocalic position alone processed the initial and final components of intervocalic place cues.

On the other hand, if the initial and final components of place of articulation information in VCV stimuli are processed by different detectors, then there will be no additivity of adaptation effects and the partial and full cue adapters should have the same effect.

It is also possible that the full cue adapters will have a smaller effect than the partial cue adapters if the direction of the F2 and F3 transitions is the critical acoustic characteristic. According to this line of reasoning, the $F 2$ and $F 3$ transitions on the VC portion are processed as if they were initial transitions and, since the direction of the VC F2 and F3 transitions for $[\mathrm{b}]$ is the same as the direction of the CV transitions for [d] (and vice versa), the VC transitions of the full cue adapters will have an adapting effect that is opposite in direction to the CV transitions (see Pisoni, Note 6, for the only report of such a finding with speech stimuli).

\footnotetext{
Method

Stimuli

The test series consisted of eight five-formant synthetic speech stimuli, ranging from [aba] to [ada], which were generated using Klatt's (1972) synthesis program. The members of the series differed in the F2 and F3 transition on the second vowel. The first part of a stimulus consisted of a 190-msec steady-state [a] with formant frequencies of $720,1,240,2,500,3,500$, and $4,500 \mathrm{~Hz}$ for $F 1$ through F5. This was followed by a $70-\mathrm{msec}$ silent closure interval, which was followed by a $250-\mathrm{msec} \mathrm{CV}$. The first formant of the $\mathrm{CV}$ had a $25-\mathrm{msec}$ transition with a starting frequency of $200 \mathrm{~Hz}$ for all stimuli. The F2 and F3 transitions were $40 \mathrm{msec}$ long, with starting frequencies of $1,000 \mathrm{~Hz}$ for $\mathrm{F} 2$ and $2,100 \mathrm{~Hz}$
}

for $F 3$ for the endpoint [aba], and 1,700 and $2,800 \mathrm{~Hz}$ for $F 2$ and $F 3$, respectively, for the endpoint [ada]. The partial cue adapters, A/BA and A/DA, were the endpoint stimuli of the series. The CV part of the full cue adapters was identical to the corresponding part of the partial cue adapters; the first part of these adapters, however, was not steady state, but had F1, F2, and $F 3$ transitions which mirror-imaged the $\mathrm{CV}$ transitions. The vowel [a] was used in this experiment rather than [ae] because the difference between the vowel formant frequencies and the F2 and F3 transitions appropriate to [b] and [d] made it unlikely that the vowel would have a [d]-like adapting effect.

\section{Subjects}

Ten M.I.T. students and employees served as subjects.

\section{Design and Procedure}

Construction of the stimulus tapes. Four unadapted identification tapes were made. The format of the tapes was identical to those of Experiments 1 and 2. One adaptation tape was made for each of the adapters. The sequence of events on each set of four trials was the same as in the previous experiments, with the exception that the adapter was repeated 40 times, rather than 45. This change was made in order to fit all the trials for one adaptation condition on a single tape.

Testing procedure. The procedure was the same as in Experiment 1 . For this experiment, the " $B$ " end of the rating scale was " 1 " and the "D" end was "8."

\section{Results \\ Effects of the Adapters on the Stimulus Series}

The average ratings under the four adaptation conditions and the unadapted identification condition are shown in Figure 5. A two-way analysis of variance for the factors adapter (A/BA, A/DA, ABBA, ADDA, and none) and stimulus ( 0 through 7) was done in order to assess the effects of the different adapters on identification of the stimuli. All factors were significant: adapter, $F(4,36)=$ 76.33, stimulus, $F(7,63)=205.25$, and the interaction, $F(28,252)=12.62, \mathrm{p}<.001$. The post hoc tests of difference between means indicated that the mean rating with both the full and partial cue [b] adapters was higher (more "D"-like) than the unadapted identification; with both [d] adapters, the mean rating was lower than with no adapter, $p<.001$. There were no differences between either the two [b] adapters or the two [d] adapters. The post hoc analysis of the interaction revealed the mean rating with A/BA to be higher than in the unadapted identification condition for Stimuli 3, 4, and 5; with ABBA, the mean rating was higher for Stimuli 2 through $5, p<.05$ or more. For both [d] adapters, the mean rating was more " $B$ "-like for Stimuli 3 through $6, p<.05$ or more. The only significant difference within a phonetic class was for Stimulus 3, for which the mean rating was higher with A/BA than with ABBA, $\mathrm{p}<.05$.

\section{Phonetic Boundaries}

The mean phonetic boundaries as determined by linear interpolation were: 3.17 for $\mathrm{A} / \mathrm{BA}, 3.17$ for ABBA, 4.81 for A/DA, and 4.91 for ADDA, and 


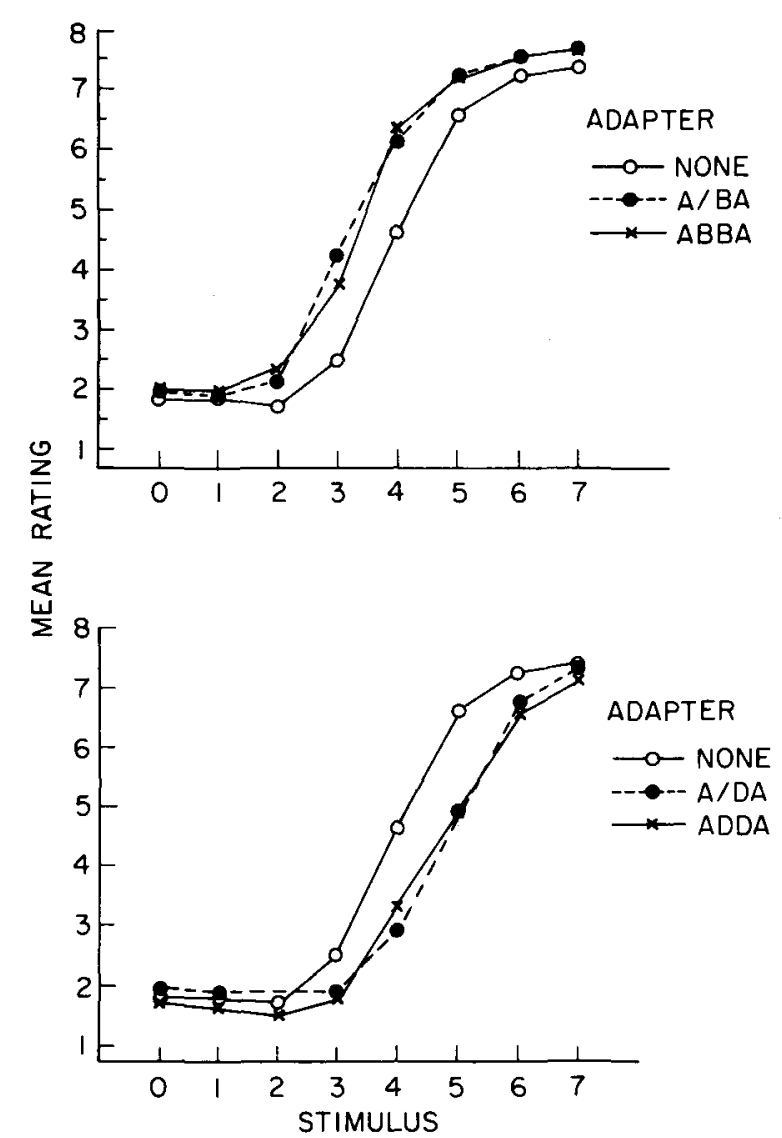

Figure 5. Mean ratings in the different adaptation conditions are shown as a function of the stimulus for Experiment 3. On both graphs, the unadapted identification condition is represented by the unfilled circles, the partial cue adapters by the filled circles, and the full cue adapter by the crosses.

3.99 in the unadapted identification condition. The analysis of variance revealed the adapter factor to be highly significant, $F(4,36)=120.62, p<.001$. The difference between the boundaries with the [b] adapters and the unadapted identification condition were highly significant, as were the differences between the boundaries with the [d] adapters and with no adapter. No differences within a phonetic class were significant.

\section{Discussion}

The finding of no difference between the effects of the full and partial cue adapters suggests that the initial and final components of place of articulation information in VCVs are processed by different detectors even when the information is embedded in a VCV context. The unitary consonant precept based on the initial and final components of intervocalic consonants must arise above the level of selective adaptation. The results of the experiment also suggest that although it is possible that detectors for place of articulation operate on general properties, such as the spectrum in the vicinity of closure, they appear to be tuned exclusively to either onset or offset characteristics.

\section{GENERAL DISCUSSION}

It appears that the answer to the queston of how listeners perceive the same stop consonant in different syllable positions is not to be found using the selective adaptation paradigm. The three experiments reported herein indicate that the invariant perception of place or articulation in different positions is not based on processes tapped by selective adaptation.

Although this study was not intended to address the issue of the auditory vs. phonetic basis of selective adaptation, a brief discussion of the nature of the selective adaptation effect seems to be in order. It is becoming increasingly evident that selective adaptation affects auditory or psychoacoustic processes. Much of the early work which was explained in terms of phonetic or linguistic feature detectors can be explained equally well in terms of the auditory characteristics of the stimuli (Ades, 1976), and other studies (Ganong, 1976; Pisoni \& Tash, 1975; Bailey, Note 7, Note 8; Sawusch, Note 1) compel an auditory interpretation of their findings. The finding of the present study-that a vowel may have an adaptation effect on a consonant if it is acoustically similar to the consonant, whereas a phonetically identical consonant which is acoustically different will not have an effect-also indicates that selective adaptation operates at a level below that at which even the basic consonant-vowel distinction is made. The fact that the question concerning the basis of the perceptual invariance for initial and final stops cannot be answered with the selective adaptation suggests that such invariance does not occur at a strictly auditory level.

Two other lines of research converge on this conclusion. Shattuck and Klatt (1976) used brief tone bursts which resembled the second and third formant transitions of $[b, d, g]$ in initial and final position in order to determine whether the mirror-image patterns were perceived as similar. They found that such patterns were perceptually less similar than patterns representing different places of articulation. In a study of infant speech perception, Williams (Note 9) found that infants of several months could make the [d]-[g] distinction in initial position, but not in postvocalic position. Thus, it appears that when perception must have a nonlinguistic basis, initial and final stops have different perceptual properties.

One interesting question raised by these findings is the question of how children come to classify stop consonants in initial and final position into the same category. The evidence from studies of selective 
adaptation suggests that this generalization is not based solely on the auditory similarity of pre- and postvocalic stops. One alternative is that this generalization is based on learning. In natural running speech, one is infrequently confronted with a CV or $\mathrm{VC}$ in isolation. More often, a consonant in running speech is embedded between two vowels. Thus, initial and final place of articulation cues are presented together. As suggested by Shattuck and Klatt (1976), a learned association may develop over time between the initial and final components of place of articulation information due to their contiguity in VCVs. An investigation of the ability of very young infants to categorize consonants according to place of articulation while ignoring syllable position would help to provide an answer to the question.

\section{REFERENCE NOTES}

1. Sawusch, J. R. The structure and flow of information in speech perception: Evidence from selective adaptation of stop consonants. Research on Speech Perception, Department of Psychology, Indiana University, August, 1976, Technical Rep. No. 2.

2. Bush, M. A., \& Williams, K. L. Infant place discrimination with and without release bursts. Paper presented at the 93rd meeting of the Acoustical Society of America, University Park, Pa., June 1977.

3. Fischer-Jorgensen, E. Identification of unreleased Danish stop consonants. Annual Report of the Institute of Phonetics, University of Copenhagen, 1972, 75-175.

4. Blumstein, S. E., \& Stevens, K. N. Perceptual invariance and onset spectra for stop consonants in different vowel environments. Paper presented at the 92nd meeting of the Acoustical Society of America, San Diego, November 1976.

S. Stevens, K. N. The potential role of property detectors in the perception of consonants. Quarterly Progress Report, Research Laboratory of Electronics, July 1973, 110, 155-168.

6. Pisoni, D. B. Stages of processing in speech perception: Feature analysis. Paper presented at the Eighth International Congress of Phonetic Sciences, Leeds, England, August 1975.

7. Bailey, P. Perceptual adaptation for acoustical features in speech perception. Speech Perception, Progress Report of the Department of Psychology, The Queens University of Belfast, 1973, $2.2,29-34$

8. Bailey, P. Preliminary to an account of formant transition perception. Speech Perception, Progress Report of the Department of Psychology, The Queens University of Belfast, 1975, 2.4, 55-59.

9. Williams, L. The effects of phonetic environment and stress placement on infant discrimination of the place of stop consonant articulation. Paper presented at the Second Annual Boston University Conference on Language Development, September 30October 1, 1977

\section{REFERENCES}

Ades, A. E. How phonetic is selective adaptation? Experiments on syllable position and vowel environment. Perception \& Psychophysics, 1974, 16, 61-66.

ADEs, A. E. Adapting the property detectors for speech perception In R. Wales \& E. Walker (Eds.), New approaches to language mechanisms. New York: North-Holland, 1976.

Butumstein, S. E., Stevens, K. N., \& Nigro, G. N. Property detectors for bursts and transitions in speech perception. Journal of the Acoustical Society of America, 1977, 61, $1301 \cdot 1313$
COOPER, W. E. Adaptation of phonetic feature analyzers for place of articulation. Journal of the Acoustical Society of America, 1974, 56, 617-627.

Cooper, W. E. Selective adaptation to speech. In F. Restle, R. M. Shiffrin, N. J. Castellan, H. Lindman, \& D. B. Pisoni (Eds.), Cognitive theory (Vol. I). Hillside, N.J: Erlbaum, 1975.

Delattre, P. C., Liberman, A. M., \& Cooper, F. S. Acoustic loci and transitional cues for consonants. Journal of the Acoustical Society of America, 1955, 27, 769-773.

DiefI, R. L. The effect of selective adaptation on the identification of speech sounds. Perception \& Psychophysics, 1975, 17, 48-52.

Eimas, P. D., \& Corbix, J. D. Selective adaptation of linguistic feature detectors. Cognitive Psychology, 1973, 4, 99-109.

GANONG, W. F., III Amplitude contingent selective adaptation to speech. Journal of the Acoustical Society of America, 1976, 59, 526. (A)

Halle, M., Hughes, G. W., \& Radley, J. P. A. Acoustic properties of stop consonants. Joumal of the Acoustical Society of America, 1957, 29, 107-116.

KLATT, D. H. Acoustical theory of terminal-analog speech synthesis. In Proceedings of the 1972 International Conference of Speech Communication and Processing, Boston, 1972.

KLATT, D. H. Voice onset time, frication and aspiration in wordinitial consonant clusters. Joumal of Speech and Hearing Research, 1975, 18, 686-706.

liberman, A. M., Cooper. F. S., Shankweiler, D. P., \& Studdert-Kennedy, M. Perception of the speech code. Psychological Review, 1967, 74, 431-461.

LINDQuist, E. F. Design and analysis of experiments in psychology and education. Boston: Houghton Mifflin, 1953.

Malecot, A. The role of releases in the identification of released final stops. Language, 1958, 34, 370-380.

MiLleR, J. L. Properties of feature detectors for speech: Evidence from the effects of selective adaptation on dichotic listening. Perception \& Psychophysics, 1975, 18, 389-397.

Mille R, J. L., \& Enmas, P. D. Studies on the selective tuning of feature detectors for speech. Journal of Phonetics, 1976, 4. 119-127.

Minifie, F., Rudegair, R., Mirstein, R., \& Vivion, M. Relative perceptual cue strength for voiced stop consonants. Journal of the Acoustical Society of America, 1971, 49, 86. (A)

Pisoni, D. B., \& TASH, J. Auditory property detectors and processing place features in stop consonants. Perception \& Psychophysics, 1975, 18, 401-408.

SAWUSCH, J. R. Selective adaptation effects on end-point stimuli in a speech series. Perception \& Psychophysics, 1976, 20, 61-65.

Shattuck, S. F., \& KLATT, D. H. The perceptual similarity of mirror-image patterns in speech. Perception \& Psycophysics, $1976,20,470-474$.

W AJSKOP, M., \& SwEERTs, J. Voicing cues in oral stop consonants. Journal of Phonetics, 1973, 1, 121-130.

WANG, W. S.-Y. Transition and release as perceptual cues for final plosives. Journal of Speech and Hearing Research, 1959, 2, 66-73.

\section{NOTES}

1. The place of articulation of a stop consonant is the point at which the vocal tract is completely occluded in the production of the consonant; [b] has a bilabial place of articulation, [d] alveolar, and [g] velar.

2. Eimas and Corbit (1973) originally proposed that these detectors had a phonetic, rather than an auditory, basis; since then, a number of researchers have presented evidence suggesting that such detectors are sensitive to auditory features rather than, or in addition to, phonetic features. The general description of the selective adaptation process given here is equally applicable to detectors based on auditory or phonetic features. 
3. This method of manipulating the burst was necessitated by the constraints of the synthesis program. There is evidence which indicates that the closure interval in final position and voice onset time in initial position are longer for [g] than for [d] (Klatt, 1975; Wajskop \& Sweerts, 1973).

4. Boundaries were also determined by first fitting a straight line to the middle five points of the series; these boundaries were essentially identical to those reported here.

5. This experiment also included two VC-like adapters, for which a cross-position effect was found. These VC-like stimuli had second- and third-formant transitions that were identical to
[Ad], but a first formant transition that was either flat or rising. Similar findings were also reported by Pisoni and Tash (1975). Although these stimuli are speech-like in some respects, they are definitely not speech. Sawusch (Note 1) attributed his findings for the VC-like adapters to peripheral, frequency-specific auditory processes. The results for the speech adapters were assumed to reflect processes operating at a more central integrative level.

(Received for publication November 2, 1977; revision accepted August 3, 1978.) 